\title{
Effekte schulischer Berufswahlaktivitäten auf die berufliche Selbstwirksamkeit von Jugendlichen beim Übergang in die berufliche Grundbildung
}

\author{
Markus P. Neuenschwander und Jan Hofmann \\ Pädagogische Hochschule FHNW Windisch
}

\begin{abstract}
Lehrpersonen können den Übergang in die berufliche Grundbildung von Jugendlichen im Rahmen von unterrichtsinternen Aktivitäten, unterrichtsexternen Aktivitäten und Coaching unterstützen. Ein Analysemodell wurde anhand einer Längsschnittstichprobe mit 1171 Jugendlichen überprüft (Befragung im 9. Schuljahr und im ersten Jahr der beruflichen Grundbildung). Ergebnisse von Strukturgleichungsmodellen zeigen, dass unterrichtsinterne Aktivitäten die berufliche Selbstwirksamkeit vor dem Übergang, das Vorwissen über den Lehrbetrieb und indirekt die berufliche Selbstwirksamkeit nach dem Übergang vorhersagen. Die Daten liefern Hinweise zu einem empirisch gestützten Modell der schulischen Unterstützung beim Übergang in die berufliche Grundbildung.
\end{abstract}

\section{Einle itung ${ }^{1}$}

Der Übergang von der Schule in die Berufsbildung ist für Jugendliche eine anspruchsvolle Entwicklungsaufgabe. Berufswahl und Integration in den beruflichen Kontext sind zwei wichtige Elemente des Übergangs. Gelingen sie, so steigen die Chancen eines Abschlusses einer Sekundarstufe II-Ausbildung (Neuenschwander et al., 2012). In der Schweiz besuchen rund zwei Drittel der Jugendlichen eine berufliche Grundbildung (Babel \& Lagana, 2016). Aufgrund der damit verbundenen hohen Bedeutung der beruflichen Grundbildung wurde im Schweizer Lehrplan 21 der Auftrag an die Lehrpersonen der Sekundarstufe I erweitert, Jugendliche auf die Berufsbildung vorzubereiten (Schweizerische Konferenz der kantonalen Erziehungsdirektoren [EDK], 2006). Empirisch überprüfte Konzepte zur Berufsvorbereitung der Schule sind sehr selten. Diese sind jedoch für eine pädagogische Theorie der schulischen Laufbahn, aber auch für die Aus- und Weiterbildung von Lehrpersonen wichtig. Zudem fehlen Studien zur Thematik, wie sich die Unterstützung der Berufswahl durch Lehrpersonen der Sekundarstufe I auf die Anpassungsprozesse nach dem Eintritt in die berufliche Grundbildung auswirken. Die Forschung zur Berufswahl vor dem Übergang (z.B. Lent \& Brown, 2013) und zur beruflichen Sozialisation nach dem Übergang in die Berufsbildung (z.B. Saks et al., 2007) sind weitgehend voneinander entkoppelt. Es ist wichtig, diese zwei Forschungstraditionen zu verbinden, weil die Berufswahl die berufliche Sozialisation im Betrieb wesentlich vorbereitet (Neuenschwander \& Hofmann, 2021).

Die EDK formulierte das Ziel für die Schweiz, dass 95\% der Jugendlichen einen Abschluss auf Niveau Sekundarstufe II erreichen, weil dadurch Ausbildungskosten und das Arbeitslosigkeitsrisiko reduziert werden (EDK, 2006). Die berufliche Selbstwirksamkeit ist eine wichtige Bedingung für den erfolgreichen Ausbildungsabschluss (Samuel \& Burger, 2020). Sie wird von den Jugendlichen im Laufe der Berufswahl entwickelt und nach dem Übertritt in die berufliche Grundbildung angepasst. Die berufliche Selbstwirksamkeit im Lehrbetrieb hängt einerseits von Ergebnissen der Berufswahl wie Vorwissen über den Lehrbetrieb oder berufliche Selbstwirksamkeit vor Beginn der Lehre ab. Andererseits wird sie auch von betrieblichen Sozialisationstaktiken zur Einführung der Jugendlichen nach dem Übertritt beeinflusst (z.B. Kammeyer-Mueller \& Wanberg, 2003).

Im Anschluss an die EDK-Richtlinie zum Erreichen eines Abschlusses auf Niveau Sekundarstufe II erhielt die schulische Berufsorientierung im Schweizer Lehrplan 21 mehr Gewicht. Es gibt aber kaum Forschung zum Thema, wie Berufswahlaktivitäten von Lehrpersonen im Zusammenspiel mit der beruflichen Selbstwirksamkeit und dem Vorwissen über den Lehrbetrieb vor dem Übertritt die berufliche Selbstwirksamkeit nach dem Übertritt vorhersagen. Solche Studien erlauben Aussagen zur längerfristigen Wirksamkeit von Berufswahl-

1 Wir danken dem Staatssekretariat für Bildung, Forschung und Innovation (Vertragsnummer: 1315000504) für die Finanzierung dieser Studie. 
aktivitäten von Lehrpersonen im Wechselspiel mit individuellen Berufswahlmerkmalen und bieten eine Hilfestellung für die Organisation des schulischen Unterrichts zur beruflichen Orientierung. Daher wird im vorliegenden Beitrag die Frage bearbeitet, wie Strategien von Lehrpersonen der Sekundarstufe I vor dem Übergang sowie von Lehrbetrieben mit der beruflichen Selbstwirksamkeit im Lehrbetrieb zusammenhängen.

Zur Beantwortung dieser Frage wird ausgehend von der sozial-kognitiven Laufbahntheorie des Selbstmanagements (Lent \& Brown, 2013) und der sozial-kognitiven Laufbahntheorie der Arbeitszufriedenheit (Lent \& Brown, 2008) ein Konzept der schulischen Berufsorientierung zum Unterricht in der Sekundarstufe I überprüft. Die beiden Theorien werden für die vorliegende Analyse zusammengeführt. In Konsequenz werden Bedingungen der Berufswahl vor dem Übergang mit Prozessen der beruflichen Anpassung nach dem Übergang verbunden, um Aspekte des Übergangs von der Schule in die berufliche Grundbildung zu erklären. Es wird anhand von Längsschnittdaten gezeigt, wie sehr von Lehrpersonen initiierte Berufswahlaktivitäten die Selbstwirksamkeit und das Vorwissen über den Lehrbetrieb vor dem Übertritt und indirekt die berufliche Selbstwirksamkeit nach dem Übergang in die Berufsbildung vorhersagen. Zudem wird im Vergleich dazu die Bedeutung der betrieblichen Sozialisationstaktiken für die berufliche Selbstwirksamkeit nach dem Übergang analysiert.

\section{Theoretischer Ansatz und Forschungsstand}

\section{Anpassungsprozesse nach Eintritt in die berufliche Grundbildung}

Lent und Brown (2008) entwickelten die sozial-kognitive Laufbahntheorie der Arbeitszufriedenheit (SCCT), der zufolge Jugendliche auf der Basis von Zielen und kontextuellen Ressourcen bzw. Hindernissen Selbstwirksamkeitserwartungen sowie Erwartungen an die zukünftige Ausbildung aufbauen. Diese Erwartungen beeinflussen, wie gut Lernende die Arbeitsziele im Betrieb erreichen, was sich in der Arbeitszufriedenheit und schliesslich in der Lebenszufriedenheit zeigt. Die Arbeitszufriedenheit und die Lebenszufriedenheit werden nach der Theorie zudem von Persönlichkeitsmerkmalen des Jugendlichen beeinflusst. Die Annahmen der Theorie wurden durch viele Studien und Metaanalysen gestützt (Brown \& Lent, 2019; Sheu et al., 2020). Angewendet auf die berufliche Grundbildung repräsentiert die berufliche Selbstwirksamkeit, ähnlich wie die Arbeitszufriedenheit, ein Erfolgskriterium für einen gelungenen Start in die Berufsbildung (Häfeli \& Schellenberg, 2009). Die berufliche Selbstwirksamkeit bezeichnet die subjektive Überzeugung, zukünftige berufliche Herausforderungen meistern zu können (Bandura et al., 2001). Die berufliche Selbstwirksamkeit und die Arbeitszufriedenheit hängen miteinander zusammen (Schyns \& Collani, 2001). Die berufliche Selbstwirksamkeit stellt eine zentrale Ressource für das Erreichen des Ausbildungsabschlusses und die Planung einer weiterführenden Ausbildung nach Abschluss der beruflichen Grundbildung dar. Die berufliche Selbstwirksamkeit am Ende des ersten Ausbildungsjahres sagt das Abbruchrisiko bzw. die Abschlusswahrscheinlichkeit der Sekundarstufe II-Ausbildung vorher (Peguero \& Shaffer, 2015; Samuel \& Burger, 2020). Sie schützt vor emotionalem Ausbrennen (Clauss et al., 2021) und erlaubt Vorhersagen, in welches Berufsfeld junge Menschen eintreten (Bandura et al., 2001; Lent et al., $2008 \mathrm{a}$ ).

Im Berufswahlprozess setzen sich Jugendliche gemäss Lent und Brown (2013) mit den beruflichen Anforderungen auseinander und entwickeln die Erwartung, wie gut sie die zukünftigen beruflichen Anforderungen erfüllen können. Diese berufliche Selbstwirksamkeit vor Lehrbeginn sagt die berufliche Selbstwirksamkeit nach dem Übergang vorher, weil sie ein über die Zeit relativ stabiles Konstrukt darstellt (Lent et al., 2008b).

Die berufliche Selbstwirksamkeit nach dem Übertritt wird aber auch durch Ergebniserwartungen bestimmt (Lent \& Brown, 2008). Ergebniserwartungen an den Lehrbetrieb bezeichnen die auf Vorwissen über den Lehrbetrieb beruhende Einschätzung der zukünftigen Situation im Lehrbetrieb. Jugendliche mit umfassendem Vorwissen haben realistische Erwartungen an die Ausbildung und sind in der Lage zu antizipieren, welche Bedingungen sie konkret vorfinden werden (Breaugh \& Mann, 1984). Dadurch können sie sich nach Eintritt in den Lehrbetrieb situationsangemessener verhalten. Sie können ihre beruflichen Ziele effizienter verfolgen und haben dadurch mehr Erfolgserfahrungen, was sich in einer höheren beruflichen Selbstwirksamkeit niederschlägt (Lent et al., 2008b).

Neben der beruflichen Selbstwirksamkeit vor Lehrbeginn und dem Vorwissen über den Lehrbetrieb wird die berufliche Selbstwirksamkeit nach Lehrbeginn gemäss dem Modell von Lent und Brown (2008) auch von proximalen Kontextfaktoren bestimmt. In der beruflichen Grundbildung wirken proximale Kontextfaktoren in den Betrieben, in den Berufsfachschulen und in überbetrieblichen Kursen. Weil die betriebliche Ausbildung den grössten Zeitanteil der Ausbildung erhält (Stalder \& Nägele, 2011), wird der Fokus auf die betriebliche Ausbildung gelegt. Die inhaltsbezogenen Sozialisationstaktiken der Betriebe zählen zu den besonders wichtigen proximalen Kontexteinflüssen (Neuenschwander \& Hofmann, 2021). Wenn die eintretenden Lernenden 
gemäss diesen Strategien in die neuen Abläufe und in das Team eingeführt werden, wenn sie bei der Arbeit konkret angeleitet werden und strukturierte Vorgänge vorfinden, so haben sie mehr arbeitsbezogenes Wissen, welches das Sammeln von Erfolgserlebnissen wahrscheinlicher macht und die berufliche Selbstwirksamkeit der Lernenden stärkt. Dies wurde in verschiedenen Studien gezeigt (Kammeyer-Müller \& Wanberg, 2003; Saks et al., 2007). Zusammenfassend werden folgende Hypothesen zum Anpassungsprozess nach dem Übergang in die berufliche Grundbildung formuliert:

Die berufliche Selbstwirksamkeit in der beruflichen Grundbildung hängt positiv zusammen mit

(H1a) den inhaltsbezogenen Sozialisationstaktiken gegenüber neu eintretenden Lernenden im Lehrbetrieb,

(H1b) dem Vorwissen der Jugendlichen über den Lehrbetrieb und

(H1c) der beruflichen Selbstwirksamkeit vor Ausbildungsbeginn.

Berufswahlaktivitäten von Lehrpersonen in der Sekundarstufe I

Nach der sozial-kognitiven Theorie des Selbstmanagements von Lent und Brown (2013) können eine hohe berufliche Selbstwirksamkeit vor Lehrbeginn und umfangreiches Vorwissen über den Lehrbetrieb als Ergebnisse der Auseinandersetzung mit der eigenen Laufbahnentscheidung verstanden werden. In der ersten Berufswahl sind die Berufswahlaktivitäten der Jugendlichen eine wichtige Form dieser Auseinandersetzung. Im Sinne eines proximalen Kontextfaktors beeinflussen Lehrpersonen durch das Initiieren entsprechender Berufswahlaktivitäten den Laufbahnentscheid der Jugendlichen und erhalten dadurch die Funktion einer sozialen Ressource. Gemäss Neuenschwander und Schaffner (2010) bezeichnen die Jugendlichen ihre Lehrpersonen nach den Eltern als zweitwichtigste Bezugspersonen im Berufswahlprozess. Eine hohe Lehrpersonenunterstützung sagt die Entwicklung einer hohen beruflichen Selbstwirksamkeit vorher (Gushue \& Witson, 2006).

Berufswahlaktivitäten von Lehrpersonen verfolgen gemäss dem Deutschschweizer Lehrplan 21 unter anderem die Ziele, die Jugendlichen zur Auseinandersetzung mit der Berufsbildung zu motivieren, Ausbildungsoptionen zu explorieren und sich auf die spezifischen Herausforderungen im Lehrstellenmarkt sowie auf den Eintritt in die berufliche Grundbildung vorzubereiten. Es gibt konzeptuelle und didaktische Arbeiten zur Unterstützung der Berufswahl in der Schule (z.B. Jung, 2020; Frank, 2020). Diese Konzepte wurden aber empirisch nicht überprüft. In einer früheren Studie (Neuenschwander \& Schaffner, 2010) wurden auf der Basis von Interviews mit Lehrpersonen schulische Berufswahlaktivitäten in drei Bereiche strukturiert. Die drei Bereiche beziehen sich auf von Lehrpersonen initiierte Berufswahlaktivitäten für ihre Schülerinnen und Schüler

(1) im Rahmen des Unterrichts im Klassenzimmer (Beispiel: Bewerbungsgespräche üben),

(2) ausserhalb des Klassenzimmers (Beispiel: einen Betrieb besichtigen) oder

(3) durch Coaching einzelner Schülerinnen und Schüler.

Berufswahlaktivitäten im Unterricht erlauben Schülerinnen und Schülern, die eigenen Interessen, Fähigkeiten, Vorbilder zu reflektieren, Strategien in der Berufswahl und für den Lehrstellenmarkt zu entwickeln und sich über berufliche und ausbildungsbezogene Optionen zu informieren (Jung, 2020). Berufswahlaktivitäten ausserhalb des Unterrichts erlauben Beobachtungen und direkte Begegnungen in möglichen Berufsfeldern und Betrieben (Lipowski et al., 2020). Im Coaching können Lehrpersonen für Jugendliche, deren Berufswahlprozess stockt oder die unzureichende Elternunterstützung erhalten, gezielte individualisierte Unterstützung leisten (Pool Maag, 2008; Stein, 2020). Es wird ausgehend von Lent und Brown (2013) postuliert, dass Lehrpersonen über geplante und durchgeführte Berufswahlaktivitäten die Auseinandersetzung der Schülerinnen und Schülern mit ihrer eigenen Berufswahl erhöhen. Die Jugendlichen lernen ihre beruflichen Fähigkeiten besser kennen und stärken ihr Wissen über den Lehrbetrieb. Zusammenfassend werden folgende Hypothesen zu Berufswahlaktivitäten von Lehrpersonen und zur beruflichen Selbstwirksamkeit nach dem Übergang in die Berufsbildung formuliert:

H2: Unterrichtsinterne Berufswahlaktivitäten von Lehrpersonen sagen ( $\mathrm{H} 2 \mathrm{a})$ die berufliche Selbstwirksamkeit vor Lehrbeginn und (H2b) das Vorwissen über den Lehrbetrieb positiv vorher. (H2c) Die berufliche Selbstwirksamkeit vor Lehrbeginn und das Vorwissen über den Lehrbetrieb mediieren den positiven Effekt dieser Berufswahlaktivitäten auf die berufliche Selbstwirksamkeit nach dem Übergang.

H3: Unterrichtsexterne Berufswahlaktivitäten von Lehrpersonen sagen (H3a) die berufliche Selbstwirksamkeit vor Lehrbeginn und (H3b) das Vorwissen über den Lehrbetrieb positiv vorher. (H3c) Die berufliche Selbstwirksamkeit vor Lehrbeginn und das Vorwissen über den Lehrbetrieb mediieren den positiven Effekt dieser Berufswahlaktivitäten auf die berufliche Selbstwirksamkeit nach dem Übergang. 
H4: Coaching sagt (H4a) die berufliche Selbstwirksamkeit vor Lehrbeginn und (H4b) das Vorwissen über den Lehrbetrieb positiv vorher. (H4c) Die berufliche Selbstwirksamkeit vor Lehrbeginn und das Vorwissen über den Lehrbetrieb mediieren den positiven Effekt dieser Berufswahlaktivitäten auf die berufliche Selbstwirksamkeit nach dem Übergang.

\section{Me thode}

\section{Stichprobe}

Die Hypothesen wurden mit Daten des Längsschnittprojekts Wirkungen der Selektion «WiSel» überprüft. Im Projekt wird der Bildungsverlauf Jugendlicher aus vier Deutschschweizer Kantonen (Aargau, Basel-Land, Bern und Luzern) von der Primarstufe in die Erwerbstätigkeit untersucht. Daten liegen aus fünf Erhebungswellen vor. Die vorliegende Analyse verwendet die Fragebogendaten der Befragung im 9. Schuljahr (Frühling 2016) und im 1. Jahr nach Schulaustritt (Frühsommer 2017). In der ersten dieser beiden Wellen $\left(\mathrm{t}_{1}\right)$ wurden die Jugendlichen entweder im Klassenverband oder privat befragt $(N=2376)$. Alle Jugendlichen, die an der ersten Erhebung teilgenommen hatten, wurden per E-Mail für eine weitere Teilnahme angefragt. Von den 2376 Jugendlichen der ersten Welle nahmen 808 auch an der zweiten Welle teil (Rücklaufquote: 34\%). In dieser Studie wurde eine Teilstichprobe von 1171 Jugendlichen untersucht, die in der ersten Welle im Klassenverbund befragt wurden und in der ersten oder zweiten Welle angaben, eine berufliche Grundbildung in einem Lehrbetrieb zu absolvieren $\left(M_{\text {Alter t1 }}=15.7, S D_{\text {Alter } \mathrm{t} 1}=0.8\right.$, Anteil weibliche Jugendliche $\left.=43 \%\right)$. Von dieser Teilstichprobe hatten 330 Jugendliche in beiden Wellen teilgenommen.

\section{Vorgehen}

Im neunten Schuljahr leiteten die Lehrpersonen der teilnehmenden Klassen die Befragungen im Klassenverbund auf der Basis einer ausführlichen Instruktion. Ein Jahr später füllten die teilnehmenden Jugendlichen standardisierte Online-Fragebogen zu Hause aus. Mitglieder des Forschungsteams baten alle Jugendlichen, die nicht an der zweiten Erhebung teilgenommen hatten, um ein Computer Assisted Telephone Interview (CATI) mit reduziertem Fragekatalog des Online-Fragebogens. Von diesen Jugendlichen füllte ein Teil später einen Ergänzungsfragebogen mit den restlichen Fragen des Online-Fragebogens aus. Im Gegensatz zur ersten Erhebung erhielten die Jugendlichen vor der zweiten Erhebung einen Einkaufsgutschein im Wert von Fr 10.für ihre bisherige Teilnahme an der Studie. Bei beiden Erhebungen wurden alle forschungsethischen und datenschutzrechtlichen Richtlinien eingehalten.

\section{Variablen}

\section{Welle 1 (letztes Schuljahr der Sekundarstufe I)}

Jugendliche berichteten ihre wahrgenommenen Berufswahlaktivitäten der Lehrperson im Klassenkontext anhand von zwölf Items (Neuenschwander \& Schaffner, 2011 und Eigenentwicklung). Die Jugendlichen schätzten die Häufigkeit der von der Lehrperson in den letzten drei Jahren durchgeführten Berufswahlaktivitäten auf einer 5-stufigen Skala ein (0 'nie', 1 ' 1 Mal', 2 ' 2 Mal', 3 ' 3 Mal', 4 ' mehr als 3 Mal'). Eine explorative Faktorenanalyse ergab zwei Faktoren. Der erste Faktor misst die von den Jugendlichen wahrgenommenen unterrichtsinternen Berufswahlaktivitäten der Lehrperson und besteht aus fünf Items $(N=1163, M=2.2, S D=0.9$, fehlende Werte $=1 \%)$. Ein Beispielitem lautete: «Fragebogen zu eigenen Interessen ausfüllen lassen» $(\alpha=.67)$. Der zweite Faktor misst die von den Jugendlichen wahrgenommenen unterrichtsexternen Berufswahlaktivitäten der Lehrperson und besteht aus sieben Items $(N=1154, M=1.0, S D=0.7$, fehlende Werte $=2 \%)$. Ein Beispielitem lautete: «Berufsinformationszentrum BIZ besucht» $(\alpha=.76)$.

Das von den Jugendlichen wahrgenommene Coaching durch die Lehrpersonen wurde mit fünf Items von Dietrich und Kracke (2009) gemessen. Die Jugendlichen schätzten Aussagen auf einer Likert-Skala von 1 'stimmt überhaupt nicht' bis 6 'stimmt voll und ganz' ein $(N=1155, M=3.4, S D=1.3$, fehlende Werte $=1 \%)$. Ein Beispielitem lautete: «Diese Lehrperson hat mir bei der Suche einer geeigneten Berufslehre bzw. weiterführenden Schule geholfen» $(\alpha=.89)$.

Die Jugendlichen berichteten ihr Vorwissen über den Lehrbetrieb anhand von fünf Items von Breaugh und Mann (1984). Die Jugendlichen schätzten Aussagen auf einer Likert-Skala von 1 'stimmt überhaupt nicht' bis 6 'stimmt voll und ganz' ein $(N=988, M=5.1, S D=0.6$, fehlende Werte $=16 \%)$. Ein Beispielitem lautete: «Ich kenne die Vor- und Nachteile meines Lehrbetriebs» $(\alpha=.84)$ 
Die berufliche Selbstwirksamkeit vor Lehrbeginn der Jugendlichen wurde anhand von sechs Items aus Rigotti et al. (2008) erhoben. Bei der Itemformulierung wurde berücksichtigt, dass die Jugendlichen ihre berufliche Grundbildung noch nicht begonnen hatten. Die Jugendlichen schätzten Aussagen auf einer Likert-Skala von 1 'stimmt überhaupt nicht' bis 6 'stimmt voll und ganz' ein $(N=1098, M=4.5, S D=0.7$, fehlende Werte $=6 \%)$. Ein Beispielitem lautete: "Was auch immer in meinem Berufsleben passieren wird, ich werde schon klarkommen» $(\alpha=.88)$.

\section{Welle 2 (1. Jahr der beruflichen Grundbildung)}

Die von den Jugendlichen wahrgenommenen inhaltsbezogenen Sozialisationstaktiken des Lehrbetriebs wurden mit drei Items aus Cable und Parsons (2001) gemessen. Am Ende des ersten Jahres der beruflichen Grundbildung schätzten die Jugendlichen retrospektiv Aussagen zum Beginn ihrer beruflichen Grundbildung auf einer Likert-Skala von 1 'stimmt überhaupt nicht' bis 6 'stimmt voll und ganz' ein $(N=281, M=4.6, S D=1.1$, fehlende Werte $=76 \%$ ). Ein Beispielitem lautete: «Zu Beginn der Berufslehre waren die einzelnen Ausbildungsschritte in meinem Lehrbetrieb klar vorgegeben» $(\alpha=.82)$.

Die berufliche Selbstwirksamkeit nach Lehrbeginn der Jugendlichen wurde mit jenen sechs Items aus Rigotti et al. (2008) erhoben, welche bereits in Welle 1 verwendet wurden. Die Jugendlichen schätzten Aussagen auf einer Likert-Skala von 1 'stimmt überhaupt nicht' bis 6 'stimmt voll und ganz' ein $(N=281, M=4.5, S D=0.7$, fehlende Werte $=76 \%$ ). Ein Beispielitem lautete: "Was auch immer in meinem Berufsleben passiert, ich werde schon klarkommen» $(\alpha=.82)$.

\section{Analysestrategie}

Zuerst wurden deskriptive Auswertungen und bivariate Korrelationsanalysen (Umgang mit fehlenden Werten: pairwise deletion) mit SPSS 25 gerechnet. Zur Prüfung der Hypothesen wurde ein Strukturgleichungsmodell (SEM) in Mplus 8.2 (Muthén \& Muthén, 1998-2017) spezifiziert und berechnet. Zur Kontrolle von Mehrebeneneffekten in den wahrgenommenen Berufswahlaktivitäten der Lehrperson im Klassenkontext wurde das SEM nach Schulklassen geclustert berechnet. Fehlende Werte wurden mittels des Full-InformationMaximum-Likelihood-Verfahrens (FIML) behandelt. Verwendet wurde der Maximum-Likelihood Schätzer mit robusten Standardfehlern (MLR). Für die Bewertung der Modell-Angemessenheit wurden folgende Anpassungsmasse verwendet: $\chi^{2}$-Statistik, Comparative Fit Index (CFI), Root Mean Square Error of Approximation (RMSEA). Die Beurteilung einer akzeptablen Modellpassung basierte auf folgenden Kriterien: CFI $\geq .95$ und RMSEA < .08 (Schreiber et al., 2006).

\section{Ergebnisse}

\section{Deskriptive Statistik}

In Tabelle 1 werden die durchschnittlichen Häufigkeiten der unterrichtsinternen und unterrichtsexternen Aktivitäten der Lehrperson sowie deren Coachingaktivitäten zur Berufsorientierung zwischen dem siebten und dem neunten Schuljahr dargestellt. Die Jugendlichen berichteten im neunten Schuljahr, dass sie die meisten der unterrichtsinternen Berufswahlaktivitäten durchschnittlich ein bis zwei Mal gezeigt hatten. Die meisten der unterrichtsexternen Berufswahlaktivitäten wurden von den Lehrpersonen durchschnittlich einmal durchgeführt. Ein ambivalentes Bild zeigt sich bezüglich der von den Jugendlichen eingeschätzten Coachingaktivitäten der Lehrpersonen. Die Aussagen dazu wurden durchschnittlich mit einer Antwort zwischen 'stimmt eher nicht' und 'stimmt eher' beurteilt. Die wahrgenommene Häufigkeit der von den Lehrpersonen durchgeführten Berufswahl- und Coachingaktivitäten weicht zwischen den einzelnen Jugendlichen stark ab (hohe Standardabweichung). 


\section{Tabelle 1}

Deskriptive Statistiken zu den Berufswahlaktivitäten

\begin{tabular}{|c|c|c|c|}
\hline Konstrukt und Items & $N$ & $M$ & $S D$ \\
\hline \multicolumn{4}{|l|}{ Unterrichtsinterne Berufswahlaktivitäten der Lehrperson } \\
\hline Das Schreiben einer Bewerbung behandelt/geübt & 1154 & 2.77 & 1.23 \\
\hline Berufswahltagebuch führen lassen & 1157 & 2.40 & 1.51 \\
\hline Das Thema Vorstellungsgespräche behandelt/geübt & 1147 & 2.27 & 1.27 \\
\hline Fragebogen zu eigenen Interessen ausfüllen lassen & 1143 & 2.04 & 1.15 \\
\hline Ablauf des Berufswahlprozesses vorgestellt (Fahrplan) & 1146 & 1.69 & 1.26 \\
\hline \multicolumn{4}{|l|}{ Unterrichtsexterne Berufswablaktivitäten der Lehrperson } \\
\hline Betriebe/Firmen besucht/besichtigt & 1158 & 1.23 & 1.36 \\
\hline Berufsinformationszentrum BIZ besucht & 1150 & 1.18 & 0.82 \\
\hline Berufstätige Personen in die Schule eingeladen & 1156 & 1.14 & 1.13 \\
\hline Elternabende zur Berufswahl durchgeführt & 1137 & 1.13 & 0.88 \\
\hline Lernende aus der Berufsbildung eingeladen (rent a stift o.ä.) & 1151 & 0.85 & 0.95 \\
\hline Projekte/Anlässe zur Berufswahl mit anderen Klassen durchgeführt & 1127 & 0.84 & 1.03 \\
\hline An einer Projekt- oder Studienwoche zum Thema Berufs- oder Studienwahl teilgenommen & 1121 & 0.56 & 0.90 \\
\hline \multicolumn{4}{|l|}{ Coaching } \\
\hline \multicolumn{4}{|l|}{ Diese Lehrperson... } \\
\hline ... hat mir bei der Suche einer geeigneten Berufslehre bzw. weiterführenden Schule geholfen. & 1151 & 3.31 & 1.60 \\
\hline ...hat mich auf verschiedene Studien- oder Berufsmöglichkeiten aufmerksam gemacht. & 1151 & 3.47 & 1.54 \\
\hline $\begin{array}{l}\text {...hat mit mir über die Chancen gesprochen, in verschiedenen weiterführenden Schulen bzw. } \\
\text { Berufslehren einen Platz zu bekommen. }\end{array}$ & 1143 & 3.31 & 1.59 \\
\hline $\begin{array}{l}\text {...hat mich ermuntert, Informationen über interessante Berufe bzw. weiterführende Schulen } \\
\text { zu suchen. }\end{array}$ & 1144 & 3.47 & 1.56 \\
\hline $\begin{array}{l}\ldots \text { hat mit mir über meine Interessen und Fähigkeiten in Bezug auf meine zukünftige Berufs- } \\
\text { lehre bzw. weiterführende Schule gesprochen. }\end{array}$ & 1132 & 3.69 & 1.52 \\
\hline
\end{tabular}

\section{Korrelationen}

Tabelle 2 zeigt, dass die berufliche Selbstwirksamkeit nach Lehrbeginn $\left(\mathrm{t}_{2}\right)$ mit der beruflichen Selbstwirksamkeit vor Lehrbeginn $\left(\mathrm{t}_{1}\right)$, dem Vorwissen über den Lehrbetrieb und den inhaltsbezogenen Sozialisationstaktiken des Lehrbetriebs signifikant korreliert $(.21<r<.27)$. Die berufliche Selbstwirksamkeit vor Lehrbeginn und das Vorwissen über den Lehrbetrieb korrelieren signifikant mit den unterrichtsinternen und -externen Berufswahlaktivitäten der Lehrperson und teilweise auch mit dem Coaching durch die Lehrperson $(.04<r<.14)$. Die unterrichtsinternen und -externen Berufswahlaktivitäten sowie Coaching durch die Lehrperson korrelieren untereinander signifikant $(.34<r<.40)$.

\section{Tabelle 2}

Interkorrelationsmatrix

\begin{tabular}{|c|c|c|c|c|c|c|c|c|c|}
\hline & $N$ & $M$ & $S D$ & 1 & 2 & 3 & 4 & 5 & 6 \\
\hline 1. UI BWA t $t_{1}$ & 1163 & 3.24 & 0.85 & - & & & & & \\
\hline 2. UE BWA $t_{1}$ & 1154 & 1.99 & 0.66 & $.38^{* * *}$ & - & & & & \\
\hline 3. Coaching $t_{1}$ & 1155 & 3.44 & 1.29 & $.40^{* * *}$ & $.34^{* * *}$ & - & & & \\
\hline 4. Berufl SWE $\mathrm{t}_{1}$ & 1098 & 4.54 & 0.72 & $.13^{* * *}$ & $.11^{* * *}$ & $.14^{* * *}$ & - & & \\
\hline 5. Vorwissen LB $t_{1}$ & 988 & 5.10 & 0.64 & $.10^{* *}$ & $.11^{* * *}$ & .04 & $.34^{* * *}$ & - & \\
\hline 6. Inhbez So $\mathrm{T}_{2}$ & 281 & 4.59 & 1.10 & $.13^{*}$ & .10 & .05 & .10 & .08 & - \\
\hline 7. Berufl SWE $t_{2}$ & 281 & 4.50 & 0.65 & .02 & -.02 & .03 & $.26^{* * *}$ & $.21^{* * *}$ & $.27^{* * *}$ \\
\hline
\end{tabular}

Legende: UI: Unterrichtsinterne, BWA: Berufswahlaktivitäten, UE: Unterrichtsexterne, Berufl SWE: Berufliche Selbstwirksamkeit, LB: Lehrbetrieb, Inhbez SoT: Inhaltsbezogene Sozialisationstaktiken des Lehrbetriebs, $\mathrm{t}_{1}$ : 9 . Schuljahr, $\mathrm{t}_{2}$ : 1. Lehrjahr, $N=251-1151$; ${ }^{* * *}: p<.001$ (zweiseitig), ${ }^{* *}: p<.01$ (zweiseitig), ${ }^{*}: p<.05$ (zweiseitig) 
Vorhersage der beruflichen Selbstwirksamkeit am Ende des ersten Lehrjahres Zur Prüfung der formulierten Hypothesen wurde ein Strukturgleichungsmodell berechnet. Die Ergebnisse werden mittels standardisierter Koeffizienten $\beta$ beschrieben. Das Modell erreichte eine gute Passung zu den Daten $\left(\chi^{2}=938.3, \mathrm{df}=603, p<.001, \mathrm{CFI}=.97\right.$, RMSEA $\left.=.02, N=1171\right)$ und ist in Abbildung 1 dargestellt.

In Übereinstimmung mit den Hypothesen $\mathrm{H} 1 \mathrm{a}, \mathrm{H} 1 \mathrm{~b}, \mathrm{H} 1 \mathrm{c}$ hing die berufliche Selbstwirksamkeit nach Lehrbeginn signifikant positiv von der beruflichen Selbstwirksamkeit vor Lehrbeginn $(\beta=.20, p=.012)$, vom Vorwissen über den Lehrbetrieb $(\beta=.20, p=.014)$ und von den inhaltsbezogenen Sozialisationstaktiken des Lehrbetriebs $(\beta=.30, p<.001)$ ab.

Die berufliche Selbstwirksamkeit vor Lehrbeginn $\left(\mathrm{t}_{1}\right)$ wurde durch die unterrichtsinternen Berufswahlaktivitäten der Lehrperson $(\beta=.11, p=.036)$ und deren Coaching $(\beta=.08, p=.039)$ signifikant positiv vorhergesagt (H2a, H4a bestätigt). Die unterrichtsexternen Berufswahlaktivitäten der Lehrperson hatten keinen Einfluss auf die berufliche Selbstwirksamkeit vor Lehrbeginn $(\beta=.03, p=.291$; H3a falsifiziert). Das Vorwissen über den Lehrbetrieb wurde durch die unterrichtsinternen $(\beta=.13, p=.044)$ und unterrichtsexternen Berufswahlaktivitäten der Lehrperson $(\beta=.10, p=.037)$ signifikant positiv vorhergesagt (H2b, H3b bestätigt). Das Coaching durch die Lehrperson hatte keinen signifikanten Einfluss auf das Vorwissen über den Lehrbetrieb $(\beta=-.04$, $p=.233$; H4b falsifiziert).

Die unterrichtsinternen Berufswahlaktivitäten der Lehrperson hatten einen signifikant positiven totalen indirekten Effekt auf die berufliche Selbstwirksamkeit nach Lehrbeginn, vermittelt über die berufliche Selbstwirksamkeit vor Lehrbeginn und über das Vorwissen über den Lehrbetrieb ( $\beta_{\text {Ind }}=.05, p=.029$; H2c bestätigt). Der direkte Einfluss der unterrichtsinternen Berufswahlaktivitäten auf die berufliche Selbstwirksamkeit nach Lehrbeginn war nicht signifikant $(\beta=-.05, p=.341)$. Der Einfluss der unterrichtsinternen Berufswahlaktivitäten der Lehrperson auf die berufliche Selbstwirksamkeit nach Lehrbeginn wurde folglich vollständig mediiert.

\section{Abbildung 1}

Vorhersage der beruflichen Selbstwirksamkeit nach Lehrbeginn durch betriebliche Faktoren und Berufswahlaktivitäten von Lehrpersonen (standardisierte Koeffizienten)

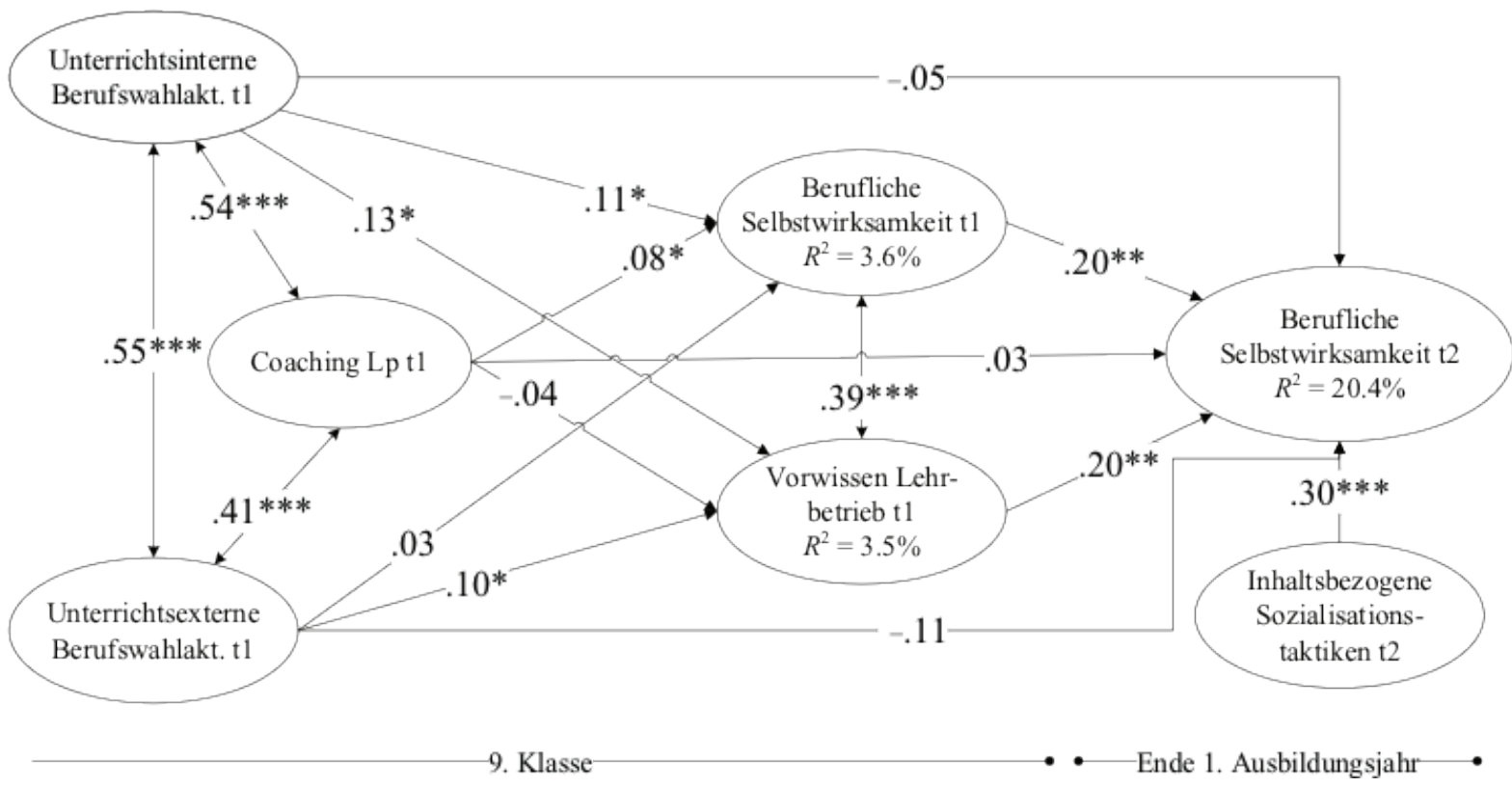

Legende: ${ }^{* * *}: p<.001,{ }^{* *}: p .<0.1, *: p<.05$ (einseitiges Signifikanzniveau)

Die unterrichtsexternen Berufswahlaktivitäten der Lehrperson und das Coaching hatten keinen totalen indirekten Effekt über die berufliche Selbstwirksamkeit vor Lehrbeginn und über das Vorwissen über den Lehrbetrieb auf die berufliche Selbstwirksamkeit nach Lehrbeginn (unterrichtsexterne Berufswahlaktivitäten: $\beta_{\text {Ind }}=.03, p=.081$; Coaching: $\beta_{\text {Ind }}=.01, p=.308 ; \mathrm{H} 3 \mathrm{c}$ und $\mathrm{H} 4 \mathrm{c}$ falsifiziert). Auch die direkten Einflüsse der unterrichtsexternen Berufswahlaktivitäten der Lehrperson und des Coachings auf die berufliche Selbstwirk- 
samkeit nach Lehrbeginn waren nicht signifikant (unterrichtsexterne Aktivitäten: $\beta=-.11, p=.200$; Coaching: $\beta=.03, p=.379$ ). Die erklärte Varianz für die berufliche Selbstwirksamkeit vor Lehrbeginn war 3.6\%, für das Vorwissen über den Lehrbetrieb 3.5\% und die berufliche Selbstwirksamkeit nach Lehrbeginn 20.4\%.

\section{Diskussion}

In diesem Beitrag wurde ein Konzept mit drei Arten von Berufswahlaktivitäten von Lehrpersonen der Sekundarstufe I vorgeschlagen und dessen Vorhersagekraft auf die berufliche Selbstwirksamkeit beim Übertritt in die berufliche Grundbildung überprüft. In Übereinstimmung mit der sozial-kognitiven Theorie der Laufbahnentscheidung von Lent und Brown $(2008 ; 2013)$ zeigen die Ergebnisse, dass häufigere Berufswahlaktivitäten von Lehrpersonen Jugendlichen helfen, vor Lehrbeginn eine hohe berufliche Selbstwirksamkeit und Vorwissen über den Lehrbetrieb zu entwickeln, welche teilweise und zusätzlich zur betrieblichen Unterstützung die berufliche Selbstwirksamkeit nach dem Übergang vorhersagen. Erstmals wurde längsschnittlich am Beispiel des Übergangs in die berufliche Grundbildung gezeigt, wie Lehrpersonen der Sekundarstufe I die Berufswahl und indirekt die berufliche Selbstwirksamkeit der Jugendlichen nach Eintritt in die Berufsbildung beeinflussen.

Während der indirekte Effekt der unterrichtsinternen Berufswahlaktivitäten auf die berufliche Selbstwirksamkeit nach dem Übergang signifikant war, waren die indirekten Effekte der unterrichtsexternen Berufswahlaktivitäten und des Coachings nicht signifikant. Wider Erwarten trägt das wahrgenommene Coaching der Lehrpersonen nicht dazu bei, dass Jugendliche mehr Vorwissen über den Lehrbetrieb haben. Die Lehrpersonen verfolgen möglicherweise mit dem Coaching primär das Ziel, die Auseinandersetzung der Jugendlichen mit den eigenen Kompetenzen und Interessen zu unterstützen (Lipowski et al., 2020; Ryter, 2014). Zudem sagen die unterrichtsexternen Berufswahlaktivitäten die berufliche Selbstwirksamkeit vor Lehrbeginn nicht vorher. Ergebnisse anderer Studien zeigten, dass unterrichtsexterne Berufswahlaktivitäten weniger auf die Interessen und Fähigkeiten des Jugendlichen fokussieren, sondern vielmehr das Erkunden von betrieblichen und beruflichen Ausbildungsbedingungen fördern (Lipowski et al., 2020). Die in dieser Studie berechneten bivariaten Korrelationen ergaben aber auch, dass die drei Arten von Berufswahlaktivitäten wesentlich miteinander zusammenhängen. Offenbar führten manche Lehrpersonen eher viele Aktivitäten durch, während andere Lehrpersonen nach allen drei Arten eher wenig Aktivitäten initiierten. In Weiterführung zur umfangreichen Forschung zu den betrieblichen Sozialisationstaktiken von jungen Mitarbeitenden (z.B. Saks \& Gruman, 2018) belegen die vorliegenden Ergebnisse auch die Bedeutung inhaltsbezogener Sozialisationstaktiken in Betrieben. Diese beschreiben formale betriebsinterne Einführungsmassnahmen wie Ausbildungskonzepte und die Strukturierung von Rückmeldungen.

Das formulierte Konzept der Berufswahlaktivitäten thematisiert sowohl Ausbildungsinhalte als auch Methoden. Neu ist, dass es empirisch den differenziellen Nutzen von Coaching sowie von unterrichtsinternen und unterrichtsexternen Berufswahlaktivitäten aufzeigt und sich nicht auf die Berufswahl beschränkt, sondern den Übergang in die Berufsbildung miteinbezieht. Es trägt damit zur Übergangsforschung bei. Bezüglich der Planung und Umsetzung der Berufswahlaktivitäten haben frühere Studien gezeigt, dass Lehrpersonen gute Beziehungen zu den Betrieben helfen. Besonders das Erkunden von Arbeitsabläufen und betrieblichen Sozialisationsprozessen der Jugendlichen ist bei Vorhandensein eines beruflichen Netzwerks einfacher möglich (Neuenschwander et al., 2016).

\section{Limitationen}

Diese Untersuchung unterliegt mehreren Limitationen. (1) Die Berufswahlaktivitäten wurden aus der Perspektive der Jugendlichen im neunten Schuljahr erfasst. Möglicherweise erinnern Jugendliche nicht alle durchgeführten Berufswahlaktivitäten. Eine mehrperspektivische Analyse unter zusätzlichem Einbezug von Lehrpersonendaten stellt daher ein wichtiges Forschungsdesiderat dar. (2) Lehrpersonen führten möglicherweise Berufswahlaktivitäten durch, die in dieser Liste nicht enthalten waren, für die Wirkung der schulischen Berufswahlaktivitäten jedoch wesentlich sind. Da die Liste auf der Basis früherer Lehrpersonenbefragungen empirisch erstellt wurde, sollten die wichtigsten Berufswahlaktivitäten jedoch enthalten sein. Bezüglich der Qualität der durchgeführten Berufswahlaktivitäten konnten keine Aussagen gemacht werden, da diese nicht erfasst wurden. Es ist anzunehmen, dass Lehrpersonen die Berufswahlaktivitäten mit unterschiedlicher Intensität umsetzen. (3) Die Zusammenhänge zwischen den Berufswahlaktivitäten und den Ergebnissen der Berufswahl wurden querschnittlich überprüft. Entsprechend sind keine kausalen Aussagen möglich. Die Berufswahlakti- 
vitäten konnten erst mit dem Abschluss des Berufswahlprozesses im neunten Schuljahr erfasst werden und das Forschungsdesign erlaubte nicht, in mehreren Zwischenerhebungen die Berufswahlaktivitäten detailliert zu erfassen. Über die Testung indirekter längsschnittlicher Effekte wurden die querschnittlich geprüften Zusammenhänge teils validiert (Einflüsse auf berufliche Selbstwirksamkeit). (4) Die Auswertung berücksichtigte zwar klassenspezifische Effekte. Allerdings wurden Differenzen in den Lehrpersonenaktivitäten zwischen den Schulformen der Sekundarstufe I (Grundansprüche, erweiterte Ansprüche, progymnasiale Ansprüche) nicht ausgewiesen. Frühere Studien berichteten Unterschiede in den Berufswahlaktivitäten von Lehrpersonen zwischen den Schulformen (z.B. Neuenschwander \& Schaffner, 2010). Zukünftige Studien sollten daher das Modell getrennt für die verschiedenen Schulformen überprüfen.

\section{Schlussfolgerungen}

Neben den betrieblichen Sozialisationstaktiken beeinflussen Ergebnisse der Berufswahl wie das Vorwissen über den Lehrbetrieb und die berufliche Selbstwirksamkeit vor Lehrbeginn kognitive Mikroprozesse der betrieblichen Anpassung von Jugendlichen (Neuenschwander \& Hofmann, 2021). Die berufliche Selbstwirksamkeit und das Vorwissen über den Lehrbetrieb beeinflussen das zielgerichtete Handeln von Lernenden im Lehrbetrieb, was sich in der beruflichen Selbstwirksamkeit nach dem Übergang zeigt.

Die Ergebnisse tragen zur Weiterentwicklung einer Didaktik der beruflichen Orientierung bei (Jung, 2020), die in der Aus- und Weiterbildung von Lehrpersonen eingesetzt werden kann. Für Lehrpersonen in der Praxis zeigt das Konzept in Verbindung mit früheren Untersuchungen (Neuenschwander, 2017) die Notwendigkeit auf, innerhalb des Unterrichts - einerseits auf Klassenebene und andererseits auf individueller Ebene - Jugendliche zur Auseinandersetzung mit der Berufswahl zu ermutigen (Neuenschwander, 2017). Die Ergebnisse differenzieren die Befunde von Gushue und Wiston (2006), wonach die Unterstützung durch Lehrpersonen positiv mit Selbstwirksamkeit und Ergebniserwartungen korreliert. Wenn Lehrpersonen die berufliche Selbstwirksamkeit von Jugendlichen erhöhen wollen, planen sie gemäss der Ergebnisse am besten unterrichtsinterne Aktivitäten wie den Ablauf des Berufswahlprozesses darstellen oder Bewerbungen und Vorstellungsgespräche üben. Sie können aber auch das Coaching mit einzelnen Jugendlichen anwenden. Zum Aufbau von Vorwissen über den Lehrbetrieb können Lehrpersonen beitragen, indem sie entweder unterrichtsinterne Aktivitäten planen oder unterrichtsexterne Aktivitäten initiieren, wie zum Beispiel Betriebe besichtigen oder das BIZ besuchen.

Aufgrund der geringen Varianzaufklärung in dieser Studie sollte die zukünftige Forschung weitere Aspekte von Lehrpersonenaktivitäten im Hinblick auf ihre Bedeutung für die Berufswahl und den Übergang in die berufliche Grundbildung identifizieren und längsschnittlich überprüfen. In diesem Bereich sind empirische Studien sehr selten. Zudem sollten Unterrichtsprozesse, welche die Berufswahl und die Anpassungsprozesse in der beruflichen Grundbildung begünstigen, detaillierter analysiert werden. Neben berufswahlbezogenen Handlungen von Lehrpersonen sagen Unterrichtsstörungen und soziale Beziehungen zwischen Lehrpersonen und Lernenden sowie zwischen den Schülerinnen und Schülern untereinander die Anpassungsprozesse nach Eintritt in die berufliche Grundbildung ebenfalls wesentlich vorher (Neuenschwander \& Gerber, 2014). Auch die Fähigkeitsselbstkonzepte von Schülerinnen und Schüler sagen die wahrgenommene Passung zwischen Person und Beruf in der beruflichen Grundbildung vorher (Neuenschwander, 2011) und sollten in zukünftigen Studien einbezogen werden. Ein vielversprechendes zukünftiges Konzept der schulischen Berufsorientierung in der Sekundarstufe I sollte daher neben berufswahlbezogenen Lehrpersonenaktivitäten auch soziale Prozesse in der Schulklasse berücksichtigen. Wichtig ist, dass das Konzept nicht nur anhand der getroffenen Berufswahl überprüft wird, sondern auch aufgrund seiner Bewährung nach Eintritt in die berufliche Grundbildung. In diesem Beitrag wurde aufgezeigt, wie eine entsprechende Umsetzung aussehen könnte.

\section{Literatur}

Babel, J., \& Lagana, F. (2016). Der Übergang am Ende der obligatorischen Schule. Bundesamt für Statistik. https://www.bfs.admin. $\mathrm{ch} / \mathrm{b} f s s t a t i c / d a m / a s s e t s / 1520326 /$ master

Bandura, A., Barbaranelli, C., Caprara, G. V., \& Pastorelli, C. (2001). Self-efficacy beliefs as shapers of children's aspirations and career trajectories. Child Development, 72(1), 187-206. https://doi.org/10.1111/1467-8624.00273

Breaugh, J. A., \& Mann, R. B. (1984). Recruiting source effect: A Test of two alternative explanations. Journal of Occupational Psychology, 57(4), 261-267. https://doi.org/10.1111/j.2044-8325.1984.tb00167.x

Brown, S. D., \& Lent, R. W. (2019). Social cognitive career theory at 25: Progress in studying the domain satisfaction and career self-management models. Journal of Career Assessment, 27(4). 1-16. https://doi.org/10.1177/1069072719852736

Cable, D. M. \& Parsons, C. K. (2001). Socialization tactics and person-organization fit. Personnel Psychology, 54(1), 1-23. https:// doi.org/10.1111/j.1744-6570.2001.tb00083.x 
Clauss, E., Hoppe, A., Schachler, V., \& O'Shea, D. (2021). Occupational self-efficacy and work engagement as moderators in the stressor-detachment model. Work \& Stress, 35(1) 1-19. https://doi.org/10.1080/02678373.2020.1743790

Dietrich, J. \& Kracke, B. (2009). Career-specific parental behaviors in adolescents' development. Journal of Vocational Behavior, 75(2), 109-119. https://doi.org/10.1016/j.jvb.2009.03.005

Frank, C. (2020). Studien- und Berufsorientierung im Fachunterricht. In S. Rahn \& T. Brüggemann (Hrsg.), Berufsorientierung - ein Lehr- und Arbeitsbuch (2. Auflage, S. 473-483). Waxmann.

Gushue, G. V., \& Whitson, M. L. (2006). The relationship among support, ethnic identity, career decision self-efficacy, and outcome expectations in African American high school students. Journal of Career Development, 33(2), 112-124. https://doi. org/10.1177/0894845306293416

Häfeli, K., \& Schellenberg, C. (2009). Erfolgsfaktoren in der Berufsbildung bei gefährdeten Jugendlichen. EDK. https://edudoc.ch/ record/35458/files/StuB29A.pdf

Jung, E. (2020). Didaktische Konzepte und methodische Zugänge der Berufs- und Studienorientierung für die Sekundarstufen I und II. In S. Rahn \& T. Brüggemann (Hrsg.), Berufsorientierung - ein Lehr- und Arbeitsbuch (2. Auflage, S. 460-472). Waxmann.

Kammeyer-Mueller, J. D., \& Wanberg, C. R. (2003). Unwrapping the organizational entry process: Disentangling multiple antecedents and their pathways to adjustment. Journal of Applied Psychology, 88(5), 779-794. https://doi.org/10.1037/00219010.88 .5 .779

Lent, R. W., \& Brown, S. D. (2008). Social cognitive career theory and subjective well-being in the context of work. Journal of Career Assessment, 16(1), 6-21. https://doi.org/10.1177/1069072707305769

Lent, R. W., \& Brown, S. D. (2013). Social cognitive model of career self-management: Toward a unifying view of adaptive career behavior across the life span. Journal of Counseling Psychology, 60(4), 557-568. https://doi.org/10.1037/a0033446

Lent, R. W., Lopez, A. M., Jr., Lopez, F. G., \& Sheu, H.-B. (2008a). Social cognitive career theory and the prediction of interests and choice goals in the computing disciplines. Journal of Vocational Behavior, 73(1), 52-62. https://doi.org/10.1016/j. jvb.2008.01.002

Lent, R. W., Sheu, H.-B., Singley, D., Schmidt, J. A., Schmidt, L. C., \& Gloster, C. S. (2008b). Longitudinal relations of selfefficacy to outcome expectations, interests, and major choice goals in engineering students. Journal of Vocational Behavior, 73(2), 328-335. https://doi.org/10.1016/j.jvb.2008.07.005

Lipowski, K., Dreer, B., Kaak, S., \& Kracke, B. (2020). Berufsfelderprobungen in der schulischen Berufsorientierung. In S. Rahn \& T. Brüggemann (Hrsg.), Berufsorientierung - ein Lehr-und Arbeitsbuch (2. Auflage, S. 446-459). Waxmann.

Muthén, L. K., \& Muthén, B. O. (1998-2017). Mplus user's guide (8th ed.). Muthén \& Muthén.

Neuenschwander, M. P. (2011). Determinanten der Passungswahrnehmung nach dem Übergang in die Sekundarstufe II. Schweizerische Zeitschrift für Bildungswissenschaften, 33(3), 401-419. https://doi.org/10.24452/sjer.33.3.4866

Neuenschwander, M. P. (2017). Was hilft bei der Berufswahl? Forschungsergebnisse zur schulischen Unterstützung von Jugendlichen. Schulmagazin 7-9, 85(4), 11-14.

Neuenschwander, M. P., \& Gerber, M. (2014). Schulische Vorbereitung auf die berufliche Sozialisation im Lehrbetrieb. Unterrichtswissenschaft, 42(3), 244-260.

Neuenschwander, M. P., \& Hofmann, J. (2021). Career Decision, Work Adjustment, and Person-Job Fit of Adolescents: Moderating Effects of Parental Support. Journal of Career Development. Advance online publication. https://doi. org/10.1177/0894845321995960

Neuenschwander, M. P., \& Schaffner, N. (2010). Berufsorientierung an Schulen (Schlussbericht). PH FHNW.

Neuenschwander, M. P., \& Schaffner, N. (2011). Individuelle und schulische Risikofaktoren und protektive Faktoren im Berufsorientierungsprozess. Deutsche Schule, 103(4), 326-340. https://www.waxmann.com/artikelART100195

Neuenschwander, M. P., Gerber, M., Frank, N., \& Rottermann, B. (2012). Schule und Beruf: Wege in die Erwerbstätigkeit. VS Verlag. https://doi.org/10.1007/978-3-531-94156-1

Neuenschwander, M. P., Rösselet, S., Cecchini, A., \& Benini, S. (2016). Unterstützung von sozial benachteiligten, bildungsfernen Eltern bei der Berufswahl Jugendlicher (Forschungsbericht Nr. 7/16). Bundesamt für Sozialversicherung (BSV). https://kjf. tg.ch/public/upload/assets/51674/Studie\%20Unterstuetzung\%20von\%20sozial\%20benachteiligten\%20bildungsfernen\%20 Eltern.pdf

Peguero, A. A., \& Shaffer, K. A. (2015). Academic self-efficacy, dropping out, and the significance of inequality. Sociological Spectrum, 35(1), 46-64. https://doi.org/10.1080/02732173.2014.978428

Pool Maag, S. (2008). Berufsintegration unter sonderpädagogischer Perspektive: Förderorientiertes Coaching von Jugendlichen am Übergang Schule - Beruf [Dissertation, Universität Zürich]. Schweizerischer Dokumentenserver Bildung. https://edudoc.ch/ record $/ 32260$ ? $\ln =\mathrm{de}$

Rigotti, T., Schyns, B., \& Mohr, G. (2008). A short version of the occupational self-efficacy scale: Structural and construct validity across five countries. Journal of Career Assessment, 16(2), 238-255. https://doi.org/10.1177/1069072707305763

Ryter, A. (2014). Jonglieren, balancieren, den Spagat wagen. In A. Ryter \& D. Schaffner (Hrsg.), Wer hilft mir, was zu werden? (S. 186-198). hep.

Saks, A. M., \& Gruman, J. A. (2018). Socialization resources theory and newcomers' work engagement. Career Development International, 23(1), 12-32. https://doi.org/10.1108/cdi-12-2016-0214

Saks, A. M., Uggerslev, K. L., \& Fassina, N. E. (2007). Socialization tactics and newcomer adjustment: A meta-analytic review and test of a model. Journal of Vocational Behavior, 70(3), 413-446. https://doi.org/10.1016/j.jvb.2006.12.004

Samuel, R., \& Burger, K. (2020). Negative life events, self-efficacy, and social support: Risk and protective factors for school dropout intentions and dropout. Journal of Educational Psychology, 112(5), 973-986. https://doi.org/10.1037/edu0000406

Schreiber, J. B., Nora, A., Stage, F. K., Barlow, E. A., \& King, J. (2006). Reporting structural equation modeling and confirmatory factor analysis results: A review. The Journal of Educational Research, 99(6), 323-338. https://doi.org/10.3200/ JOER.99.6.323-338 
Schweizerische Konferenz der kantonalen Erziehungsdirektoren [EDK]. (2006). Leitlinien zur Optimierung der Nahtstelle obligatorische Schule - Sekundarstufe II. EDK. https://edudoc.ch/record/24718? In=de

Schyns, B., \& Collani, G. v. (2001). A new occupational self-efficacy scale and its relation to personality constructs and organizational variables. European Journal of Work and Organizational Psychology, 11(2), 219-241. https://doi. org/10.1080/13594320244000148

Sheu, H. B., Lent, R., Lui, A. M., Wang, X. T., Phrasavath, L., Cho, H.-J., \& Morris, T. R. (2020). Meta-analytic path analysis of the social cognitive well-being model. Journal of Counseling Psychology, 67(6), 680-696. https://doi.org/10.1037/cou0000431

Stalder, B. E., \& Nägele, C. (2011). Vocational education and training in Switzerland: Organisation, development and challenges for the future. In M. Bergman, S. Hupka-Brunner, A. C. Keller, T. Meyer, \& B. E. Stalder (Hrsg.), Youth transitions in Switzerland: Results from the TREE panel study (S. 18-39). Seismo.

Stein, M. (2020). Von PatInnen und LotsInnen. Coaching- und Mentoringprogramme in der Studien- und Berufsorientierung. In S. Rahn \& T. Brüggemann (Hrsg.), Berufsorientierung - ein Lehr- und Arbeitsbuch (2. Auflage, S. 435-445). Waxmann.

Schlagworte: Berufliche Orientierung; Lehrpersonen; berufliche Selbstwirksamkeit; berufliche Grundbildung; Übergang Schule-Beruf

\section{Effets des activités de choix de carrière à l'école sur l'auto-efficacité professionnelle des adolescent.e.s lors de la transition vers l'EFP}

Ré sumé

Les enseignant.e.s peuvent soutenir la transition vers la formation professionnelle initiale des jeunes à l'aide d'activités au sein de la classe, d'activités extra-scolaires et d'un accompagnement. Un modèle a été testé sur la base d'un échantillon longitudinal de 1171 jeunes (enquête pendant la 9e année scolaire et la formation professionnelle initiale). Les résultats des modèles d'équations structurelles montrent que les activités au sein de la classe corrèlent avec l'auto-efficacité professionnelle, ainsi qu'avec la connaissance de l'entreprise et indirectement avec l'auto-efficacité professionnelle après la transition. Les données fournissent des indices pour un modèle empirique de soutien scolaire lors de la transition vers la formation professionnelle initiale.

Mots clé : Orientation professionnelle ; enseignant.e.s ; auto-efficacité professionnelle ; formation professionnelle initiale ; transition de l'école au travail

\section{Effetti delle attività di scelta della carriera scolastica sull'autoefficacia professionale dei giovani nella transizione verso la formazione professionale}

\section{Riassunto}

Gli insegnanti possono sostenere il passaggio alla formazione professionale di base dei giovani attraverso attività scolastiche, attività extra-scolastiche e coaching individuale. Un modello è stato testato sulla base di un campione longitudinale di 1171 giovani (indagine nel $9^{\circ}$ anno scolastico e nella formazione professionale di base). I risultati dei modelli di equazioni strutturali mostrano che le attività scolastiche correlano direttamente con l'autoefficacia professionale, e con la conoscenza dell'azienda prima della transizione, mentre predicono indirettamente l'autoefficacia professionale dopo la transizione. I dati forniscono la prova di un modello empirico di sostegno scolastico durante il passaggio alla formazione professionale di base.

Parole chiave: Orientamento professionale; insegnanti; autoefficacia professionale; formazione professionale di base; passaggio dalla scuola al lavoro 


\section{Effects of career choice activities in school on adolescents' occupational self-efficacy in the transition to VET}

\section{Abstract}

Teachers support students' transition into Vocational Education and Training (VET) by classroom activities, out-of-school activities, and coaching. A model was examined using a longitudinal sample of 1171 adolescents (surveyed in 9th grade and in first year of VET). Results from structural equation models showed that classroom activities directly correlate with occupational self-efficacy and that prior knowledge about the apprenticeship program indirectly predict occupational self-efficacy after transition to VET. The findings indicate an evidencebased model of school support during the transition from school to VET.

Keywords: Professional orientation; teachers; occupational self-efficacy; vocational education and training (VET); school-to-work transition

Markus P. Neuenschwander, Prof. Dr., Leiter Forschungszentrum Lernen und Sozialisation der PH FHNW, Dozent Univ. Basel. Themen: Übergang Schule-Beruf, Chancengerechtigkeit, Verhaltensprobleme.

Pädagogische Hochschule FHNW, Zentrum Lernen und Sozialisation, Bahnhofstrasse 6, CH-5210 Windisch E-Mail: markus.neuenschwander@fhnw.ch

Jan Hofmann, MSc, wissenschaftl. Mitarbeiter Forschungszentrum Lernen und Sozialisation der PH FHNW, Doktorand Universität Basel. Themen: Übergang Schule-Beruf mit Fokus auf explorativen Berufswahlhandlungen.

Pädagogische Hochschule FHNW, Zentrum Lernen und Sozialisation, Bahnhofstrasse 6, CH-5210 Windisch E-Mail: jan.hofmann@fhnw.ch 